(2) Open Access Full Text Article

\title{
New national Biobank of The Danish Center for Strategic Research on Type 2 Diabetes (DD2)
}

This article was published in the following Dove Press journal:

Clinical Epidemiology

14 September 2012

Number of times this article has been viewed

\author{
Henry Christensen' \\ Jens Steen Nielsen ${ }^{2}$ \\ Karina Meden Sørensen ${ }^{3}$ \\ Mads Melbye ${ }^{3}$ \\ Ivan Brandslund 1,4 \\ 'Department of Clinical Biochemistry, \\ Vejle Hospital, Vejle, Denmark; \\ ${ }^{2}$ The Danish Centre for Strategic \\ Research in Type 2 Diabetes (DD2), \\ Department of Endocrinology \\ M, Diabetes Research Centre, \\ Odense University Hospital, \\ Odense, Denmark; ${ }^{3}$ Department \\ of Epidemiology Research, Statens \\ Serum Institut, Copenhagen, Denmark; \\ ${ }^{4}$ Institute of Regional Health Research, \\ University of Southern Denmark, \\ Odense, Denmark
}

Correspondence: Henry Christensen Department of Clinical Biochemistry, Vejle Hospital Kabbeltoft 25,

DK-7I 00 Vejle, Denmark

Tel +4579406634

Fax +457940 6853

Email henry.christensen@slb.

regionsyddanmark.dk
Abstract: Long-term storage of biological samples from patients has become increasingly important in studies of disease control and treatment. The first nationwide Danish diabetes project, ie, The Danish Center for Strategic Research in Type II Diabetes (DD2), aims to improve treatment and the long-term outcome of patients with newly diagnosed type 2 diabetes (T2D). The DD2 project includes establishment of a biobank with samples from 50,000 patients with newly diagnosed T2D. This paper describes how blood and urine samples from 10,000 patients per year are collected, handled, and stored. The biobank includes whole blood, DNA, and plasma and urine samples, all frozen at $-80^{\circ} \mathrm{C}$. Sampling tubes have been standardized and are sent to hospital outpatient clinics and general practitioners where samples are taken, handled, aliquoted, and returned by mail overnight in standardized cryostorage tubes. When received at the biobank, samples are frozen without further treatment. From each patient, 24 cryostorage tubes are stored. Each tube is labeled with a barcode that links the data to other information available in a clinical databank registry. When patients are enrolled in DD2, a questionnaire is filled out and a quality monitoring system ensures that patients, samples, and questionnaires can be linked together at all times. The biobank is located at Vejle Hospital and the Danish National Biobank at Statens Serum Institut. As of the end of March 2012, samples from 1186 patients have been stored, and currently samples from 8-10 patients arrive per day. We have established the first national biobank in Denmark where blood, DNA, and plasma and urine samples from patients with newly diagnosed T2D are systematically collected and stored. This biobank enables sophisticated analysis of genetic variation and response to treatment, as well as disease marker studies that better classify disease status, progression, and complications.

Keywords: DD2, biobank, type 2 diabetes, logistics, implementation, biorepository, data registration

\section{Introduction}

The general purpose of a biobank is to collect biological samples for research that can be used to determine, for example, environmental exposure, physiologic functions, and the genetic composition and functioning of an individual. ${ }^{1}$ Furthermore, biobanks have become increasingly important in research studying new biomarkers for the prediction, diagnosis, progression, and treatment of disease.

The Danish Center for Strategic Research in Type 2 Diabetes (DD2) aims to optimize treatment of patients with type 2 diabetes (T2D) by individualizing treatment. As part of the program, biological material and clinical information on 50,000 incident patients with T2D will be collected over a 5-year period. Detailed information will be gathered on all those enrolled with respect to social characteristics, disease phenotype, 
genetics, and other biomarker characteristics. To achieve this and to study the long-term impact on outcome, there is a need to gain knowledge about patients at registration when clinical data are collected, as described by Nielsen et al, ${ }^{2}$ and to obtain biological samples to be stored in a biobank, as described in this paper. To follow clinical outcomes for this cohort of patients with newly diagnosed T2D, the registered data will subsequently be linked to different Danish populationbased registries. This will provide unique outcome data, as described by Thomsen et $\mathrm{al}^{3}$ and Beck-Nielsen et al. ${ }^{4}$ In brief, the aims of this paper are to describe the biobank logistics and how the biological samples are collected, handled, shipped, and stored.

\section{Biobank organization and logistics Organization}

According to the Danish law on biomedical research ethics, it is required that patients give their signed informed consent prior to participation in a research project and storage of personal biological samples. ${ }^{5}$ A DD2 application is sent to and approved by the Danish National Committee on Biomedical Research Ethics (record number S-20100082) and the Danish Data Protection Agency (record number 2008-58-0035) prior to collection, storage, and entry of any personalized data in the biobank and database. The diagnosis of T2D is given according to the World Health Organization ${ }^{6}$ and recommendations by the Danish National Board of Health. ${ }^{7}$

The biobank is administered by the DD2 partners listed on the http://www.dd2.nu website. Each patient may, at any time, request withdrawal from the project and, consequently, samples and data from this patient will be destroyed and the patient registered as having dropped out. Furthermore, DD2 is an open project where researchers are able to present project proposals and gain access to collected biological material and clinical data. The patients participating in the project are not given compensation or other benefits for donating blood and urine. The biobank will be located at the Laboratory Centre at Vejle Hospital (ISO 15189 accredited) and at the newly established Danish National Biobank at Statens Serum Institut. The institutions will be responsible for the compliance of the biobank with Danish law.

\section{Logistics}

The prospective DD2 project aims to improve T2D treatment, as described in detail by Steffensen et al. ${ }^{8}$ This will be done by gathering clinical and biological material nationwide from newly diagnosed T2D patients. This will only be possible if the logistics for sampling and handling are simple and can be performed at hospital outpatient clinics, by general practitioners, and at other places in the health care system where patients with T2D are encountered. This requirement is balanced by the need to retrieve samples of high quality under perfect standardized conditions. Care was therefore taken to develop a collection strategy where the highest possible sample quality is achieved, allowing nationwide sampling of different sample types. To standardize the procedure as much as possible, all materials for sampling and shipping are assembled in a DD2 sampling kit, and one is prepared for each patient to be enrolled.

A detailed description of sampling and handling was initially written and made available on the DD2 website. Later this was accompanied by video instructions for preparing the buffy coat. Following sampling and handling, the collected biological material is packed in certified packaging, contained in the kits, and mailed overnight to the biobank (see Figure 1). Most biochemical markers are stable for up to 30 hours. $^{9-11}$ At the biobank, samples are received and immediately stored at $-80{ }^{\circ} \mathrm{C}$ in freestanding freezers. Currently, the laboratory centre at Vejle Hospital has employed a specialist in bioinformatics who is responsible for the biobank database administration and a biotechnologist who is responsible for all logistics. Similar activities have been established at the Danish National Biobank, including the establishment of a Danish National Biobank Registry (http://www.nationalbiobank.dk).

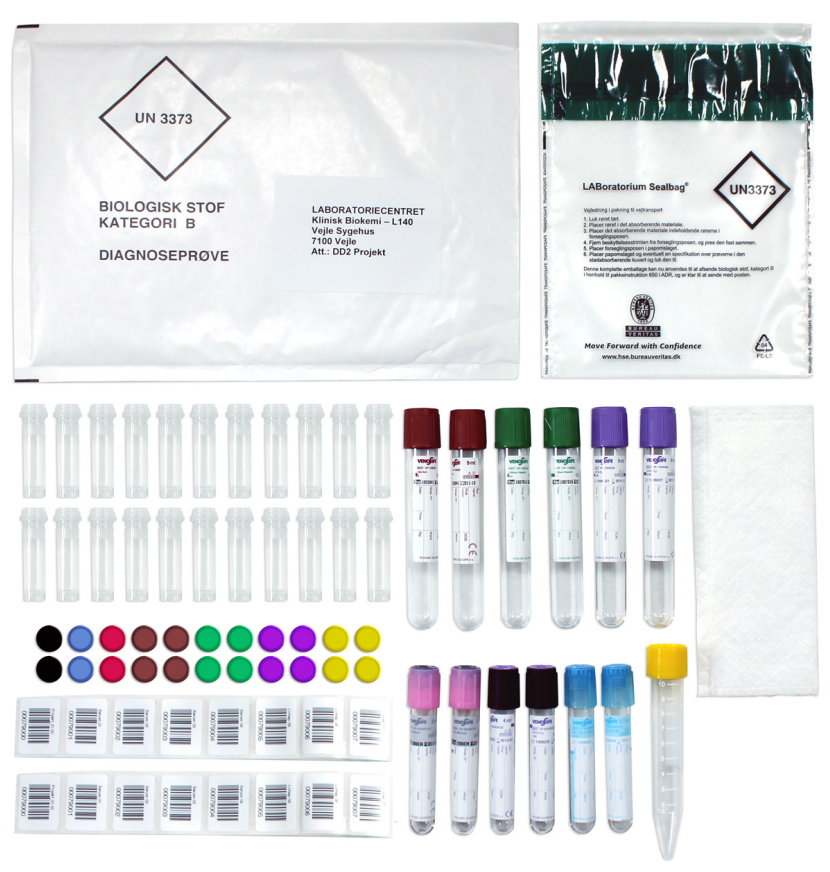

Figure I Complete sampling kit for The Danish Center for Strategic Research in Type II Diabetes. 
The above description of the DD2 biobank illustrates that the setup includes an entity that receives, stores, processes, and/or disseminates specimens, as needed, encompasses the physical location as well as the full range of operational activities, is one freezer or a separate unit or part of an institution, and has professional staff and a commitment to maintaining and preserving specimens and records for future and historical continuity. The DD2 biobank thus fulfils the definition and criteria for a biorepository. ${ }^{1,12}$

\section{Procedures for sampling and storage Sampling kit}

A DD2 sampling kit is prepared for each patient to be enrolled. Kits are packed in boxes containing 5, 10, 25, or 50 kits labeled with shelf-life expiration data and sent to the local sampling sites. Each sampling kit contains 12 primary blood sampling tubes and one tube for urine sampling. Further, it contains 22 secondary cryostorage tubes, 25 labels with barcodes, and material for packing and sending samples safely to the biobank according to Danish law (see Figure 1). The 12 primary blood sampling tubes allow a wide spectrum of different analyses to be conducted and consist of tubes with lithium heparin, sodium citrate, potassium ethylenediamine tetra-acetic acid (EDTA), sodium fluoride citrate, and clot activator (Table 1).

\section{Local procedures}

Each patient's signed informed consent to donate blood and urine to the DD2 project is checked before sampling. Prior to the sampling day, patients are informed by the local DD2 personnel to arrive in the morning after 8 hours of fasting, prior to taking morning medication, and to bring a morning urine sample. If patients forget the urine sample, a spot sample is taken on site and this is registered. Further, in case the patient is not fasting, blood samples are taken, and this is registered and information is sent with the samples to the biobank or is marked on the online registration form (for details, see Nielsen et $\mathrm{al}^{2}$ ). Fasting blood samples have been obtained from 940 of the first 1186 patients enrolled in DD2.

Blood sample tubes are always filled in the order outlined in Table 1 and each tube is carefully mixed by turning the tube upside down 10 times. At least 30 minutes and no later than 2 hours after sampling, all samples except the urine and whole blood EDTA tubes are centrifuged for 10 minutes at 1500-2000 g, and the desired content is then immediately transferred to a cap color-matching cryostorage tube using a pipette.

The 25 labels provided in the sampling kit have an eightdigit printed barcode, where the first six digits are consecutive unique numbers and the last two are a series from 01 to 24 , and they are placed on the cryostorage tubes to identify the content of each of the tubes. The last label has " 00 " as the last two digits and is used to identify and link the patient with their civil registration number on the request form that is sent to the biobank together with the samples. In this way, samples are linked to an individual personal civil registration number. The civil registration number is used nationwide to store individual personal data in Danish population-based databases, and can therefore be used when DD2 data are subsequently linked to the different Danish population-based databases, as described by Thomsen et al. ${ }^{3}$

\section{Procedures at biobank and storage}

Upon receiving the samples at the biobank they are visually inspected for volume and appearance in the form of hemolysis, possible coagulation, and buffy coat quality. Each sample is identified and registered in a database. The data shown in Table 2 are registered in this database, and each sample is assigned to a predetermined position in the

Table I List of primary sampling tubes contained in the sampling kit for The Danish Center for Strategic Research in Type II Diabetes (further details on local processing are given in the fourth column)

\begin{tabular}{|c|c|c|c|}
\hline Number of tubes & Tube volume & Additive & Processing \\
\hline 2 & $9 \mathrm{~mL}$ & Lithium heparin & Spun and plasma is aliquoted into four cryostorage tubes \\
\hline 2 & $2.7 \mathrm{~mL}$ & Citrate & Spun and plasma is aliquoted into two cryostorage tubes \\
\hline 2 & $9 \mathrm{~mL}$ & EDTA & $\begin{array}{l}\text { Spun, the plasma is aliquoted into four cryostorage tubes, and the } \\
\text { buffy coat is skimmed and aliquoted in two cryostorage tubes }\end{array}$ \\
\hline 2 & $4 \mathrm{~mL}$ & EDTA & No processing, whole blood sent in primary tubes \\
\hline 2 & $3 \mathrm{~mL}$ & Fluoride citrate & Spun and plasma is aliquoted into two cryostorage tubes \\
\hline 2 & $9 \mathrm{ml}$ & None & Spun and plasma is aliquoted into four cryostorage tubes \\
\hline I & $10 \mathrm{~mL}$ & Conical tube for urine & Urine aliquoted into four cryostorage tubes \\
\hline Total 13 & Total mL blood $=73.4$ & & Total aliquots frozen $=24$ \\
\hline
\end{tabular}

Abbreviation: EDTA, ethylenediamine tetra-acetic acid. 
Table 2 Individual information registered in the biobank database for each individual sample

\begin{tabular}{l}
\hline Database fields \\
\hline Project identification (barcodes 00-24) \\
Civil registration number \\
Serial number (determines position in freezer) \\
Sampling date \\
Freezing date \\
Sampling location \\
Fasting \\
Discrepancies \\
Comments
\end{tabular}

freezers identified in a three-dimensional system for easy later retrieval. Key information linking the barcode on the tubes in the Biobank to the patient identification is only present in the database. Thus, samples in the Biobank cannot be linked to an individual person without access to the database. The database is stored on an offline computer and protected by an access code known by two persons. A back-up of the database is performed daily. A copy of the content is also stored in a secure safe.

The freezers used operate at $-80^{\circ} \mathrm{C}$. The temperature is centrally logged for alarm purposes to ensure an emergency electrical supply. Of all samples sent to the biobank, 94\% were frozen within 30 hours after sampling. The delayed samples (6\%) are not discarded, but the delay is registered in the database. In $2.8 \%$ of the samples, the buffy coat containing the DNA material was not isolated correctly; however, DNA from all these samples has been secured by storing whole blood samples.

\section{Quality and sample limitations}

Based on experience from the diabetes biobank at Vejle where patient samples were handled and stored at $5^{\circ} \mathrm{C}$, it was initially proposed to handle and transport samples at $5^{\circ} \mathrm{C}$, but most general practitioners do not have the equipment for handling blood samples at low temperature. Establishing a nationwide transport system for blood samples at $5^{\circ} \mathrm{C}$ would also be expensive. Thus, to increase the number of sampling sites and reduce costs, all samples are taken and processed at room temperature and sent by ordinary mail.

Storing different types of plasma enables later analysis of a wide range of biomarkers and substances in the blood of patients with newly diagnosed T2D. In brief, lithium heparin tubes are used to obtain plasma for biochemistry analysis. Potassium EDTA is the standard anticoagulant used for hematology and biochemistry because it preserves the cellular components of blood. However, potassium, sodium, and some enzymes may not be measured due to addition of potassium to the sample, and because calcium and magnesium is chelated by EDTA. Sodium fluoride citrate is used for accurate glucose (and lactate) determination due to inhibition of the enzyme systems involved in glycolysis. Finally, DNA extraction and genetic analysis are possible from the separated buffy coat collected from potassium EDTA tubes or from the whole blood sample stored in EDTA tubes. Research groups can apply to the board of the DD2 partners (http://www.dd2.nu) for specimens and patient data in specific research projects.

\section{Biobank status}

The Biobank is projected to receive 50,000 patients and to store at least 1.2 million individual samples. As of March 2012, 1186 blood and urine samples have been received from 65 sampling sites located nationwide. At present, sample collection is increasing substantially (Figure 2) and the enrolment rate has increased by a factor of 5.8 since spring 2011 when the first sites started officially after a short test phase. The 1186 enrolled patients have yielded a total of 25,968 cryostorage tubes and 2268 primary sample tubes stored in the DD2 bank at present.

\section{Perspectives}

In 2009, Time magazine published a special issue on ten ideas that are changing the world, where the formation of biorepositories for research and better diagnosis and treatment of disease was one of the ten. ${ }^{13}$

The DD2 database and Biobank will enable researchers to add to the knowledge about $\mathrm{T} 2 \mathrm{D}$ and further increase the quality of medical care, both in general and for individual patients. For example, in the future, markers may be found to be predictive for the effect of a certain drug. All patients could be investigated for this trait immediately and have their treatment changed, giving the same standard of care irrespective of residence in the country.

Furthermore, new analytical tests for newly discovered biochemical or genetic components of possible importance in T2D can be tested retrospectively before being used prospectively. However, one should be aware that the nationwide multicenter approach using a practical way of collecting samples and mailing them overnight may jeopardize analyses and data acquisition for unstable or fragile metabolic or biochemical components. 


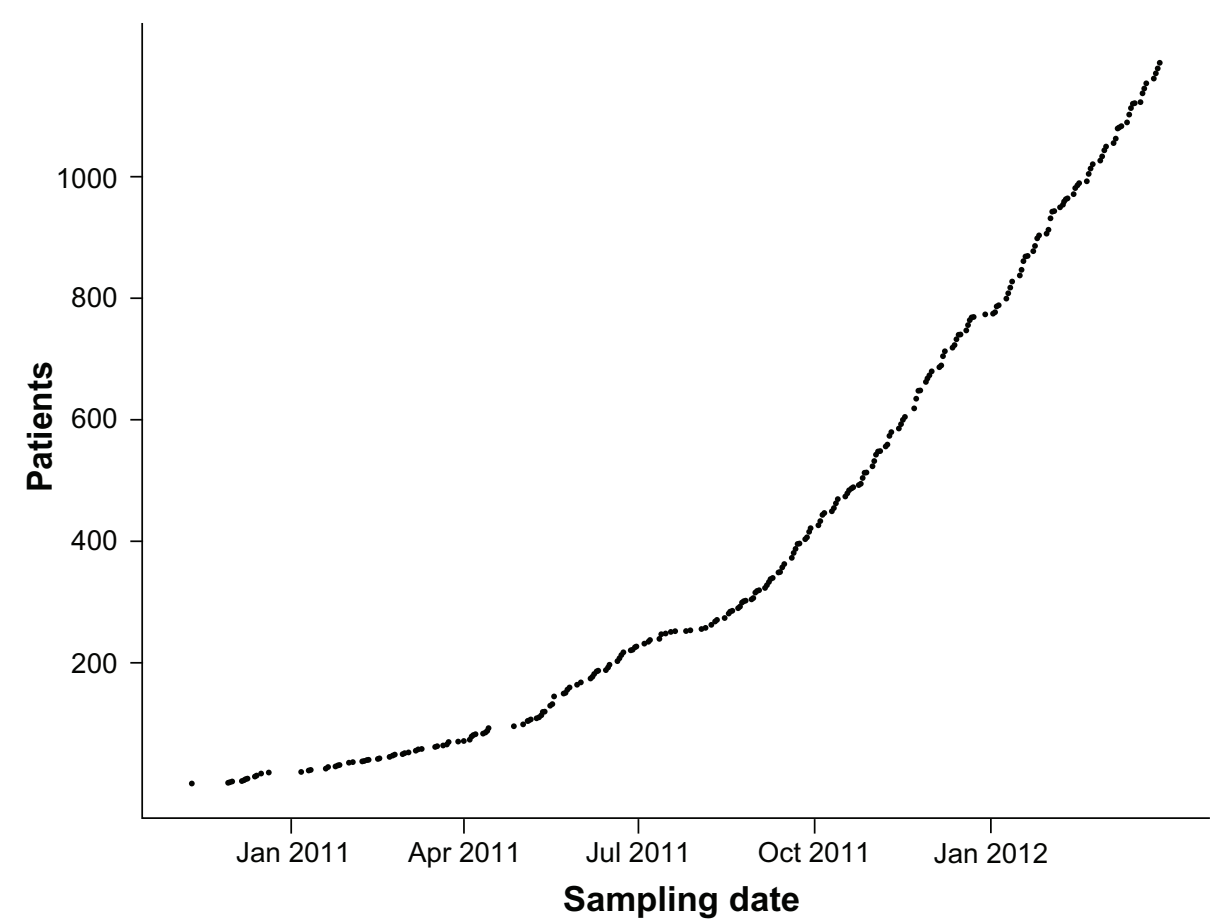

Figure 2 Accumulated number of patients from whom blood, DNA, and plasma and urine samples have been received and stored at The Danish Center for Strategic Research in Type 2 Diabetes biobank during the initial project period.

\section{Conclusion}

To the best of our knowledge, biological samples and clinical data from a large cohort of newly diagnosed patients with T2D have not previously been stored together in a biobank and database on a nationwide scale. A nationwide biobank program has been developed to store blood, DNA, and plasma and urine samples from patients with newly diagnosed T2D. A total of 1,200,000 samples from 50,000 patients will be collected and stored over a 5 -year period. As of March 2012, samples from 1186 newly diagnosed T2D patients have been received. The DD2 biobank together with the DD2 database that is linked to the different Danish population-based registries provides a unique infrastructure for studying the progression of T2D. It is our hope that this new facility will lead to new knowledge about how patient stratification can be optimized and tailored treatment implemented in the health care system, both nationally and internationally.

\section{Acknowledgment}

DD2 is the acronym for 'The Danish Centre for Strategic Research in Type 2 Diabetes' supported by the Danish Agency for Science (grant no. 09-067009 and 09-075724). DD2 is also supported by The Danish Health and Medicines Authority, The Danish Diabetes Association and an unrestricted donation from Novo Nordisk A/S. The partners of the project are listed on the project website at www. DD2.nu

\section{Disclosure}

The authors report no conflicts of interest in this work.

\section{References}

1. Ricchiuti V, Gunter EW, Vaught J. Biorepositories: Worth Their Weight in Gold. [CD-ROM] Product ID: 6634: American Association for Clinical Chemistry, Washington, DC; 2011.

2. Nielsen JS, Thomsen RW, Steffensen C, Christiansen JS. The Danish Centre for Strategic Research in Type 2 Diabetes (DD2) study: Implementation of a nationwide patient enrollment system. Clin Epidemiol. 2012:4 Suppl 1. In press.

3. Thomsen RW, Friborg S, Nielsen JS, Schroll H, Johnsen SP. The Danish Centre for Strategic Research in Type 2 Diabetes (DD2): Organization of diabetes care in Denmark and supplementary data sources for data collection among DD2 study participants. Clin Epidemiol. 2012:4 Suppl 1. In press.

4. Beck-Nielsen H, Solomon PJ, Lauridsen J, et al. The Danish Centre for Strategic Research in Type 2 Diabetes (DD2) study - expected outcome from the DD2-project and two intervention studies. Clin Epidemiol. 2012:4 Suppl 1. In press.

5. retsinformation.dk. Lov om videnskabsetisk behandling af sundhedsvidenskabelige forskningsprojekter - retsinformation.dk. (Act on Research Ethics Review of Health Research Projects). Danish. Available at: http://www.retsinformation.dk/forms/R0710.aspx?id=137674. Accessed March 13, 2012.

6. World Health Organization. Use of glycated haemoglobin (HbA1c) in the diagnosis of diabetes mellitus. Abbreviated report of a WHO consultation. 2011. Available at: http://www.who.int/diabetes/publications/ report-hba1c_2011.pdf. Accessed June 17, 2012. 
7. The Danish National Board of Health. Endret metode til at diagnosticere type 2 diabetes [Modified method for the diagnosis of Type 2 diabetes. (translation from danish)]. Available at: http://www.sst.dk/Nyhedscenter/Nyheder/2012/Diabetes2AnbefaletMetode.aspx. Accessed May 9, 2012.

8. Steffensen C, Thomsen RW, Vaag A, et al. The Danish Centre for Strategic Research in Type 2 Diabetes (DD2) Project: rationale and planned nationwide studies of genetic predictors, physical exercise and individualised pharmacological treatment. Clin Epidemiol. 2012:4 Suppl 1. In press.

9. Stahl M, Brandslund I. Controlled storage conditions prolong stability of biochemical components in whole blood. Clin Chem Lab Med. 2005;43(2):210-215.

10. Jensen EA, Stahl M, Brandslund I, Grinsted P. Stability of heparin blood samples during transport based on defined pre-analytical quality goals. Clin Chem Lab Med. 2008;46(2):225-234.
11. Clinical and Laboratory Standards Institute. H18-A4 procedures for the handling and processing of blood specimens for common laboratory tests; approved guideline - fourth edition. 2010. Available at: http://www.clsi. org/source/orders/free/h18-a4.pdf. Accessed June 17, 2012.

12. Baust JG. ISBER: Best practices for repositories and trends at the institute for problems of cryobiology and medicine. Cell Preserv Technol. 2008;6(1):1.

13. TIME.com. 10 ideas changing the world right now. Available at: http://www.time.com/time/specials/packages/article/0,28804,1884779_1884782_1884749,00.html. Accessed March 8, 2012 .
Clinical Epidemiology

\section{Publish your work in this journal}

Clinical Epidemiology is an international, peer-reviewed, open access journal focusing on disease and drug epidemiology, identification of risk factors and screening procedures to develop optimal preventative initiatives and programs. Specific topics include: diagnosis, prognosis, treatment, screening, prevention, risk factor modification, systematic

Submit your manuscript here: http://www.dovepress.com/clinical-epidemiology-journal

\section{Dovepress}

reviews, risk \& safety of medical interventions, epidemiology \& biostatical methods, evaluation of guidelines, translational medicine, health policies \& economic evaluations. The manuscript management system is completely online and includes a very quick and fair peer-review system, which is all easy to use. 